\title{
АНАЛИЗ ФАКТОРА ЗАТУХАНИЯ ИНДУКЦИОННОГО ВЛИЯНИЯ МЕТИЛЬНОЙ ГРУППЫ ПО ИЗМЕНЕНИЮ ИОНИЗАЦИОННОГО ПОТЕНЦИАЛА
}

Изменение потенциалов ионизации (ПИ) под влиянием алкильных заместителей некоторыми авторами рассматривается как индукционный эффект $\left[{ }^{1-5}\right]$. Распространение индукционного влияния через $\sigma$-связи вызывает ослабление его приблизительно одинаково на каждую промежуточную $-\mathrm{CH}_{2}$-группу [6-7]. Исходя из этой точки зрения и из анализа экспериментальных данных, было представлено уравнение, описывающее изменение ПИ $\Delta I$ при последовательном метильном замещении $\left[{ }^{1-3}\right]$ :

$$
\Delta I=K \cdot z^{n}
$$

где $K$ - коэффициент пропорциональности, $z-$ фактор затухания, т. е. постоянная, характеризующая затухание индукционного влияния на одной - $\mathrm{CH}_{2}$-группе, $n-$ число - $\mathrm{CH}_{2}$-групп между присоединяемой метильной и хромофорной группами (из последней при ионизации удаляется электрон).

Принципиальное значение в формуле (1) имеет фактор затухания $z$. Его определения через константу равновесия и константы скорости реакции вместе с анализом полученных значений $z$ приведены в работе [8]. В настоящей работе исследованы постоянство фактора затухания при изменении числа метиленовых групп и его универсальность при описании различных хромофорных групп. Существование постоянного фактора в изменении ПИ показывает, что влияние заместителя передается через углеродную цепь индукционным механизмом.

\section{Методика расчета}

Рассмотрены соединения, имеющие общую формулу

$$
\mathrm{Y}-\left(\mathrm{CH}_{2}\right)_{n}-\mathrm{X} \text {, }
$$

где $\mathrm{Y}$ - хромофорная группа, X - заместитель (при алкилзамещенных соединениях $\mathrm{CH}_{3}$-группы).

Изменения ПИ, описываемые формулой (1), представляют собой бесконечно убывающую геометрическую прогрессию с первым членом $K$ и множителем $z(0<z<1)$. Сумму этого сходящего ряда обозначим $S$

$$
S=\frac{K}{1-z}
$$


Фактор затухания, являясь множителем ряда, выражается в виде

$$
z=\frac{I_{n+1}-I_{n+2}}{I_{n}-I_{n+1}} .
$$

С увеличением числа метиленовых групп ПИ уменьшается и стремится к пределу $I_{\infty}$.

В силу свойства бесконечно убывающей геометрической прогрессии изменение ПИ в отношении предела $I_{\infty}$ также является геометрическим рядом, первым членом которого выступает $S$ и множителем $-z$. Из этого ряда для определения $\mathrm{z}$ можно вывести новую формулу

$$
z=\frac{I_{n+1}-I_{\infty}}{I_{n}-I_{\infty}} .
$$

В отношении первого члена $I_{\mathrm{CH}_{3} \mathrm{Y}}$ изменение ПИ также является рядом. Формула общего члена этого ряда

$$
I_{n}-I_{\mathrm{CH}_{3} \mathrm{Y}}=S z^{n}-S .
$$

Таким образом, для обозначения изменения ПИ имеем следующие три ряда:

$$
\begin{array}{llllll}
I_{n}-I_{n+1} & : K & K z & K z^{2} & \ldots K z^{n} & \ldots \\
I_{n}-I_{\infty} & : S & S z & S z^{2} & \ldots S z^{n} & \ldots \\
I_{n}-I_{\mathrm{CH}_{3} \mathrm{Y}}: & 0 & S(z-1) & S\left(z^{2}-1\right) & \ldots S\left(z^{n}-1\right) & \ldots
\end{array}
$$

Выбор ряда обусловливается главным образом обеспечиваемой им точностью и точностью определения ПИ исходного вещества. Так, нашример, формулы (3) и (5) по сравнению с формулами (2) и (4) обеспечивают бо́льшую точность, так как абсолютные величины изменения ПИ в случае применения формулы (3) имеют большие числовые значения, и $I_{\infty}$, по существу, является для каждой серии соединений константой.

Ряд (6) можно привести к новому виду:

$I_{n}-I_{\mathrm{CH}_{3} \mathrm{Y}}=S\left(z^{n}-1\right)=-S\left(1-z^{n}\right)=-S(1-z)\left(1+z+z^{2}+\ldots+z^{n-1}\right)$.

Так как $S(1-z)=K$, то

$$
\begin{aligned}
& I_{n}-I_{\mathrm{CH}_{3} \mathrm{Y}}=-K\left(1+z+z^{2}+\ldots+z^{n-1}\right), \\
& I_{n}=I_{\mathrm{CH}_{3} \mathrm{Y}}+K \mu \text {, }
\end{aligned}
$$

где $\mu=-\left(1+z+z^{2}+\ldots+z^{n-1}\right)$.

В выражении (8) параметр $K$ характеризует серию, а параметр $\mu$ представляет индукционное влияние алкильного заместителя подобно $\sigma^{*}$-константам Тафта. Формула (8) является удобной для корреляции величин ПИ с $\mu$-константами заместителей.

\section{Обсуждение результатов}

В табл. 1 приведены факторы затухания 1-алкенов, 1-алкинов, алкилхлоридов, алкилбромидов, алкилйодидов, альдегидов и метилкетонов. Вычисления проведены по формуле (2) на основе величин ПИ, определенных фотоионизационным способом [2,9-11]. Для 1-алкенов и 1-алкинов вычисления проведены и по формуле (3). Значением $I_{\infty}$ для 1-алкенов взят определенный в нашей лаборатории ПИ 1-нонена 9,42 0,01 эв, а для

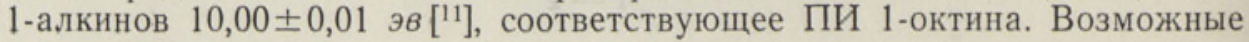




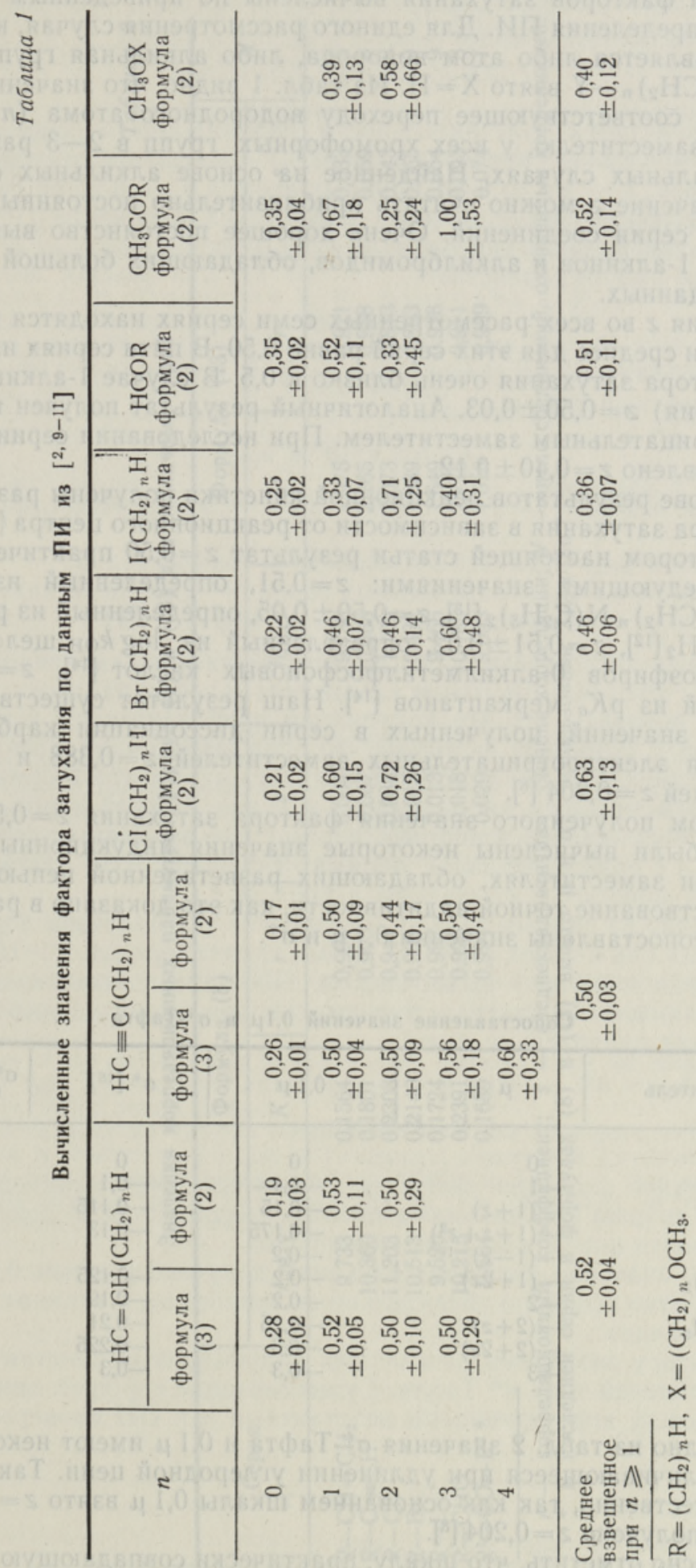


отклонения факторов затухания вычислены по приведенным величинам точности определения ПИ. Для единого рассмотрения случая, когда заместителем является либо атом водорода, либо алкильная группа, в формуле $\mathrm{X}-\left(\mathrm{CH}_{2}\right)_{n}-\mathrm{Y}$ взято $\mathrm{X}=\mathrm{H}$. Из табл. 1 видно, что значение фактора затухания, соответствующее переходу водородного атома $(n=0)$ к метильному заместителю, у всех хромофорных групп в 2-3 раза меньше, чем в остальных случаях. Найденное на основе алкильных соединений $(n \geqslant 1)$ значение $z$ можно считать приблизительно постоянным в пределах одной серии соединений. Очень хорошее постоянство выявляется у 1 -алкенов, 1-алкинов и алкилбромидов, обладающих большой точностью исходных данных.

Значения $z$ во всех рассмотренных семи сериях находятся в пределах $0,36-0,63$ и среднее для этих серий равно 0,50 . В пяти сериях из семи значение фактора затухания очень близко к 0,5 . В случае 1 -алкинов (самая точная серия) $z=0,50 \pm 0,03$. Аналогичный результат получен и в серии с электроотрицательным заместителем. При исследовании серии из диэфиров установлено $z=0,40 \pm 0,12$.

На основе результатов реакционной кинетики получены разные значения фактора затухания в зависнмости от реакционного центра $\left[{ }^{8,12}\right]$. Полученный автором настоящей статьи результат $z=0,50$ практически совпадает со следующими значениями: $z=0,51$, определенный из $\mathrm{p} K_{a}$ для серии $\left.\mathrm{NC}\left(\mathrm{CH}_{2}\right)_{n} \mathrm{~N}\left(\mathrm{C}_{2} \mathrm{H}_{5}\right)_{2}{ }^{\left[{ }^{13}\right.}\right], z=0,50 \pm 0,05$, определенный из р $K_{a}$ аминов $\mathrm{X}\left(\mathrm{CH}_{2}\right)_{n} \mathrm{NH}_{2}\left[{ }^{12}\right], z=0,51 \pm 0,02$, определенный из $\log k_{\mathrm{OH}}$ щелочного гидролиза тиоэфиров 0 -алкилметилфосфоновых кислот [14], $z=0,51 \pm 0,03$, полученный из $\mathrm{p} K_{a}$ меркаптанов [14]. Наш результат существенно отличается от значений, полученных в серии диссоциации карбоксильных кислот для электроотрицательных заместителей $z=0,388$ и алкильных заместителей $z=0,204\left[^{8}\right]$.

C учетом полученного значения фактора затухания $z=0,50$ по формуле (9) были вычислены некоторые значения индукционных постоянных $\mu$. При заместителях, обладающих разветвленной цепью, учитывалось существование точной аддитивности, как это доказано в работах $\left[{ }^{1,2}\right]$. В табл. 2 сопоставлены значения $0,1 \mu$ и $\sigma^{*}$.

Таблица 2

Сопоставление значений $0,1 \mu$ и $\sigma^{*}$-Тафта

\begin{tabular}{|c|c|c|c|c|}
\hline Заместитель & $\mu$ & $0,1 \mu$ & $\sigma^{*}\left[{ }^{15}\right]$ & $\sigma_{\text {испр. }}^{*}$ по (1 \\
\hline $\begin{array}{l}\mathrm{CH}_{3} \\
\mathrm{C}_{2} \mathrm{H}_{5} \\
\mathrm{C}_{3} \mathrm{H}_{7} \\
\mathrm{C}_{4} \mathrm{H}_{9} \\
\left(\mathrm{CH}_{2}\right)_{\infty} \mathrm{CH}_{3} \\
\mathrm{CH}_{2} \mathrm{CH}\left(\mathrm{CH}_{3}\right)_{2} \\
\mathrm{CH}_{2}\left(\mathrm{CH}_{3}\right)_{2} \\
\mathrm{CH}\left(\mathrm{CH}_{3}\right)_{2} \mathrm{C}_{5} \\
\mathrm{CH}\left(\mathrm{C}_{2} \mathrm{H}_{5}\right)_{2} \\
\mathrm{C}\left(\mathrm{CH}_{3}\right)_{3}\end{array}$ & $\begin{aligned} & 0 \\
- & 1 \\
- & (1+z) \\
- & \left(1+z+z^{2}\right) \\
- & (1-z)^{-1} \\
- & (1+2 z) \\
- & 2 \\
- & (2+z) \\
- & (2+2 z) \\
- & 3\end{aligned}$ & $\begin{array}{l}\quad 0 \\
-0,1 \\
-0,15 \\
-0,175 \\
-0,2 \\
-0,2 \\
-0,2 \\
-0,25 \\
-0,3 \\
-0,3\end{array}$ & $\begin{array}{l}\quad 0 \\
-0,1 \\
-0,115 \\
-0,13 \\
-0,125 \\
-0,19 \\
-0,21 \\
-0,225 \\
-0,3\end{array}$ & $\begin{array}{l}0 \\
-0,1 \\
-0,14 \\
-0,185 \\
-0,17 \\
-0,17 \\
-0,23 \\
-0,27 \\
-0,30\end{array}$ \\
\hline
\end{tabular}

Как видно из табл. 2 значения $\sigma^{*}$-Тафта и $0,1 \mu$ имеют некоторое различие, увеличивающееся при удлинении углеродной цепи. Такое явление вполне естественно, так как основанием шкалы $0,1 \mu$ взято $z=0,50$, а при $\sigma^{*}$ шкале получено $z=0,204\left[^{8}\right]$.

Интересно отметить, что шкалу, практически совпадающую со шкалой $0,1 \mu$, можно получить и другим путем. В работе $\left[{ }^{16}\right]$ при анализе ПИ 18 


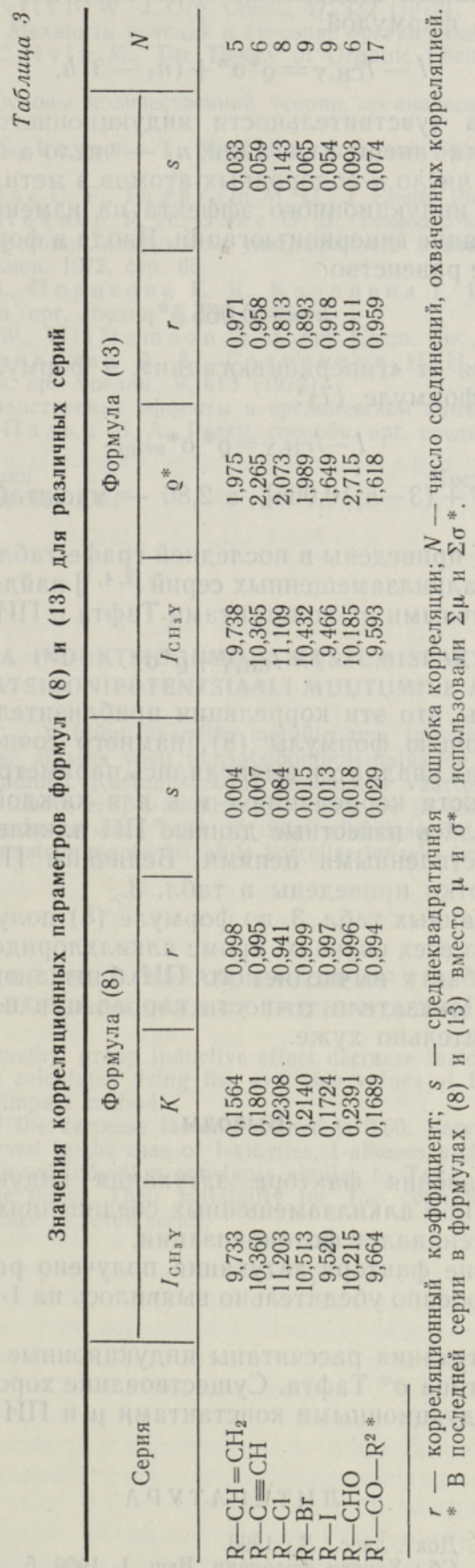


серий алкилзамещенных соединений было найдено, что изменение ПИ можно представить формулой:

$$
I-I_{\mathrm{CH}_{3} \mathrm{Y}}=\mathrm{Q}^{*} \sigma^{*}+\left(n_{1}-3\right) h,
$$

где $\varrho^{*}$ - константа чувствительности индукционного эффекта данной серии, $h$ - константа гиперконъюгации, $n_{1}-$ число $\alpha$-водородных атомов в заместителе, 3 - число $\alpha$-водородных атомов в метильной группе.

Кроме влияния индукционного эффекта на изменение ПИ получено также большое влияние гиперконъюгации. Вводя в формулу (10) найденное в той же работе равенство

$$
h=-0,065 \varrho^{*},
$$

можно освободиться от «гиперконъюгации», и формула (10) принимает вид, аналогичный формуле (7):

$$
I-I_{\mathrm{CH}_{3} \mathrm{Y}}=Q^{*} \sigma^{*}{ }_{\text {иепр, }}
$$

где $\sigma^{*}$ испр. $=2,86\left[\sigma^{*}+\left(3-n_{1}\right) 0,065\right]$, а 2,86 - масштабный коэффициент шкалы.

Значения $\sigma^{*}$ нспр. приведены в последней графе табл. 2.

Для некоторых алкилзамещенных серий $\left[{ }^{2}, 4,5\right]$ найдена линейная зависимость между обычными $\sigma^{*}$-константами Тафта и ПИ

$$
I=I_{\mathrm{CH}_{3} \mathrm{Y}}+\mathrm{Q}^{*} \sigma^{*} \text {. }
$$

Можно показать, что эти корреляции приблизительны и результаты, получаемые с помощью формулы (8), намного точнее. Для сравнения методом наименьших квадратов вычислялись параметры формул (8), (13) и показатели точности корреляции $r$ и $s$ для каждой серии. В рамках серии коррелировались известные данные ПИ алкильных соединений, в том числе и с разветвленными цепями. Величины ПИ взяты из [2,9-11]. Результаты обработки приведены в табл. 3.

Как видно из данных табл. 3, по формуле (8) получена хорошая корреляция, так как во всех случаях, кроме алкилхлоридов, $r>0,99$. Среднеквадратичная ошибка $s$ вычисляемого ПИ близка ошибке экспериментальных данных. Показатели точности корреляции по формуле (13) во всех случаях значительно хуже.

\section{Выводы}

Рассчитаны значения фактора затухания индукционного влияния метильной группы при алкилзамещенных соединениях. В основу расчета взяты разности потенциалов фотоионизации.

Среднее значение фактора затухания получено равным 0,50 , причем его постоянство особенно убедительно выявилось на 1-алкинах, 1-алкенах и алкилбромидах.

По фактору затухания рассчитаны индукционные константы алкильных заместителей типа $\sigma^{*}$ Тафта. Существование хорошей линейной корреляции между индукционными константами $\mu$ и ПИ проверено на семи сериях.

ЛИТЕРА Т У Р А

1. В и ле с о в Ф. И., Докт. дисс., Л., 1966.

2. В илесов В. И., Сб.: Успехи фотоники, Вып. 1. 1969, 5.

3. А копян М. Е., Ж. фнз. хим., 44, 2918 (1970). 
4. Levitt L. S., Levitt B. W., Chem. Ind. (London), 990 (1970).

5. L e vit t L. S., L e vit t B. W., J. Org. Chem., 37, 332 (1972).

6. Ингольд К. К., Механизм реакций и строение органических соединений, М., 1959.

7. Branch G. E., C alvin M., The Theory of Organic Chemistry, New York, 1941, p. 217

8. Па льм В. А., Основы количественной теории органических реакций. Л., 1967, c. $96-132$.

9. W a t a $n$ a be K., N a k a y a m a T., Mott 1 J., J. Quant. Spectrosc. Rad. Transfer, 2, 369 (1962).

10. Steiner B., Gi ese C. F., Inghr a m M. G., J. Chem. Phys., 34, 189 (1961).

11. Виллем Я. Я., Пи к в р Р. И., С а к с О. В., Тезисы 3-й Всесоюзной конференции по спектроскопии вакуумного ультрафиолета и взаимодейст. излуч. с веществом. Харьков, 1972, стр. 65.

12. Богатков С. В., Порисова Е. Я., Калинина Г. Р., Соколова Т. Д., Реакц. способн. орг. соедин. 5, 793 (1968).

13. Stevens on G. W., Willi a m son D., J. Am. Chem. Soc., 80, 5943 (1958).

14. Я рв Я. Л., А в икса ар А. А., Годовиков Н. Н., Морозова Н. А., Реакц. способн. орг. соедин., 9, 813 (1972).

15. Т а ф т Р., Пространственные эффекты в органической химии, гл. ХІІІ, М., 1960.

16. Пылдо я П. К., П а льм В. А., Реакц. способн. орг, соедин., 4, 786 (1967).
Ннститут физики
Академии наук Эстонской ССР
Поступила в редакцию
$22 /$ I 1974

\section{P. POLDOJA}

\section{METUULRUHMA INDUKTSIOONMÕJU KUSTUMISFAKTORI ANALUUS IONISATSIOONIPOTENTSIAALI MUUTUMISE ALUSEL}

Arvutati $\mathrm{CH}_{3}\left(\mathrm{CH}_{2}\right)_{n}$ Y tüüpi ühendite metüülrühma induktsioonmõju kustumisfaktori väärtused. Arvutuse aluseks võeti ionisatsioonipotentsiaalide muutused fotoionisatsioonimeetodil saadud andmeil. Kustumisfaktori keskmiseks väärtuseks saadi 0,50 . Kustumisfaktori konstantsus ilmnes eriti 1-alküünide, 1-alkeenide ja alküülbromiidide puhul.

Kustumisfaktori kaudu arvutati Tafti $\sigma^{*}$ tüüpi alküülasendajate induktsioonkonstandid. Nende sobivust ionisatsioonipotentsiaalide korrelleerimiseks kontralliti 7 seerial.

\section{P. POLDOJA}

\section{EXAMINATION OF THE DECREASE FACTOR OF THE METHYL GROUP INDUCTIVE EFFECT BASED ON DATA OF THE IONIZATION POTENTIAL CHANGE}

The values of the methyl group inductive effect decrease factor for compounds of the $\mathrm{CH}_{3}\left(\mathrm{CH}_{2}\right)_{n}$ Y type are calculated using the available values of the ionization potentials obtained by the photon impact method.

The mean value of the decrease factor obtained is 0.50 . Very good constancy of the decrease factor is observed in the case of 1-alkynes, 1-alkenes and alkylbromides.

The alkyl substitutional induction constants similar to Taft's $\sigma^{*}$ ones are also calculated from the decrease factor. Their suitability for the correlation of the ionization potentials has been checked in seven series. 